\title{
AChE for DNA degradation
}

\author{
Cell Research (2015) 25:653-654. doi:10.1038/cr.2015.52; published online 1 May 2015
}

\begin{abstract}
DNA hydrolysis is a biochemical process often associated with different forms of cell death, including apoptosis. In a recent paper published in Cell Discovery, Du et al. report that synaptic acetylcholinesterase (AChES) shows an unexpected enzymatic activity as DNase switched on after cytotoxic insults.
\end{abstract}

The controlled degradation of the genomic content in a cell committed to die has been the subject of intense research for decades. Several nucleases involved in DNA degradation during mammalian cell death have been identified. The nuclear DNA can be degraded either inside or outside the dying cell. The nucleases implicated in the DNA degradation inside the cell are broadly grouped as "cell-autonomous" nucleases. In later steps, DNA hydrolysis can also take place outside the cell. The endonucleases responsible for that are called "waste-management" DNases. Among the proposed roles for DNA degradation during cell death are the elimination of harmful DNAs, the amplification of death signals, the connection to DNA damage and repair pathways, the promotion of phagocytosis, and the avoidance of autoimmune diseases (reviewed in [1]).

Each form of cell death is associated with different patterns of DNA degradation, implying the activation of diverse nucleases [2]. During apoptosis, DNA degradation is a consequence of the digestion of chromatin by endogenous endonucleases that target linker DNA between the nucleosomes, yielding a characteristic pattern of low molecular weight DNA fragments known as DNA laddering. The main apoptotic nuclease is DNA fragmentation factor,
40-kDa subunit/caspase-activated DNase (DFF40/CAD), which is activated by caspases [3]. Other nucleases, such as endonuclease $\mathrm{G}$ (endoG), are also involved in apoptosis. EndoG does not discriminate between internucleosomal and nucleosomal DNA, giving a pattern of DNA degradation different from the DFF40/CAD-mediated DNA laddering [4]. In other types of non-apoptotic cell death (e.g., necrosis), proteases and DNases are released from lysosomes, leading to a smear of DNA as a result of the degradation of both DNA and DNA-bound proteins [5].

In a recent paper published in Cell Discovery, Zhang's lab unveiled an unexpected DNase activity for synaptic acetylcholinesterase (AChE-S) [6]. The $A C h E$ gene can generate three mature mRNA transcripts by alternative splicing: "synaptic" AChE-S (also known as AChE-T), "erythrocytic" AChE-E (also known as AChE-H) and "readthrough" AChE-R. AChE-S is a critical enzyme regulating neurotransmission at cholinergic synapses. Besides that, the $A C h E$ gene has also emerged as a tumor suppressor gene [7]. Du et al. [6] show that recombinant AChE-S holds intrinsic endonuclease activity, between amino acids 32-138, both in in vitro assays using plasmid DNA and in pre-fixed and detergent-permeabilized cells. However, whether AChE-S is a true apoptotic DNase in cellula needs to be further confirmed. In this sense, and unfortunately, the authors only performed TUNEL assay in AChE-Soverexpressing cells and, as recently described, TUNEL-positive cells do not necessarily undergo DNA laddering [8]. Therefore, further efforts are required to address this key issue. In any case, the identification of this new enzymatic activity of AChE-S opens a new avenue in the field of DNA degradation during physiological (e.g., for neural plasticity) and pathological cell death in, for example, cancer and neurological disorders (reviewed in [7]).

Regarding the antitumoral effects of AChE isoforms, their expression levels determine cell sensitivity towards cytotoxic agents. In this sense, different $\mathrm{AChE}$ variants are expressed in a broad battery of non-cholinergic cells as part of the cytotoxic response to various cellular insults [7]. Interestingly, in hepatocellular carcinomas, one of the most prevalent human malignancies worldwide, AChE inversely associates with tumor aggressiveness and patient prognosis [9]. In addition, Du and colleagues show that cells overexpressing nuclear-directed AChE-S impair tumorigenesis in $\mathrm{BALB} / \mathrm{c}$ nude mice. More importantly, the tumor suppressor proprieties of AChE-S rely on its DNase activity, rather than its acetylcholinesterase activity, since cells overexpressing a truncated form of AChE-S lacking the nuclease region still maintain tumorigenic proprieties when injected in nude mice.

Although preliminary, another interesting result from Du et al. relies on the nuclear translocation of AChE-S in isolated primary hippocampal neurons cultured in the presence of $\beta$-amyloid peptide (25-35). The nuclear redistribution of AChE-S correlates with TUNEL staining, which led us to speculate about the potential role of the DNase activity of AChE-S in neurodegenerative disorders, particularly in Alzheimer's disease. Therefore, it should be of special interest to explore 
more deeply the cytotoxic mechanisms of AChE-S-related DNase activity to identify potential tools of therapeutic interest, different from the current approaches used clinically by inhibiting the acetylcholinesterase-related activity of AChE-S, which targets late aspects of the disease having a minimal impact on the disorder [10].

In apoptotic cell death, AChE is necessary for apoptosome assembly and the activation of the initiator caspase-9 (reviewed in [7]; Figure 1). Interestingly, some non-cholinergic cells constitutively express different AChE variants but others do not. Therefore, a transcriptional response should exist to induce the expression of $\mathrm{AChE}$ isoforms after apoptotic stimuli. In this sense, $A C h E$ promoter contains AP1-like, ATF2 and Smad3/4 binding elements that allow transcriptional expression through c-jun, ATF2 and Smad3 [11, 12]. The inhibition of c-jun, or its upstream activating kinase, JNK, abolishes cytotoxic-triggered transcriptional upregulation of AChE [11]. Therefore, a fine control of cytotoxic-mediated upregulation of AChE could be also of special interest to manipulate undesirable cellular responses in, for example, Alzheimer's disease.

Finally, another interesting point not addressed by $\mathrm{Du}$ and colleagues concerns the potential conception of AChE-S as a "waste-management" DNase involved in non-cell autonomous DNA degradation. To date, only DNase I (as secreted nuclease) and DNase II (as lysosomal non-secreted nuclease) are considered "waste-management" DNases (reviewed in [1]). It is necessary to stress that AChEs are secreted proteins. Data provided by Zhang's lab show that

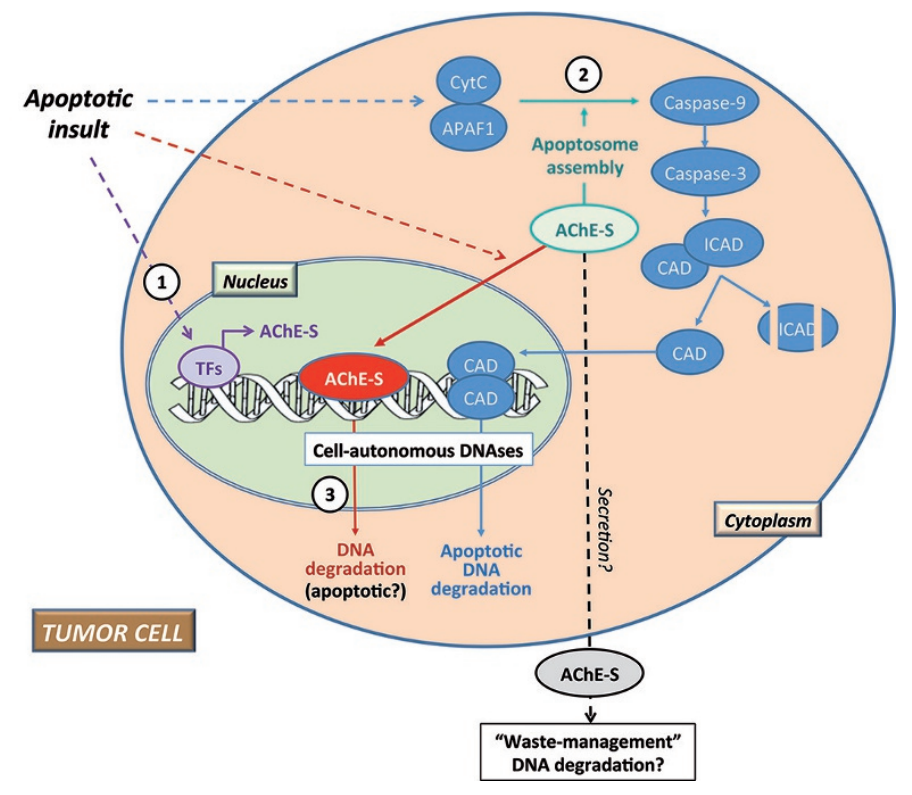

Figure 1 Schematic illustration showing the cytotoxic role of synaptic acetylcholinesterase (AChE-S) as a novel DNase. After apoptotic insult, the activation of a specific transcriptional program allows the expression of AChE-S (1). Then, AChE$S$ participates in apoptosome formation (2), promoting the activation of initiator caspase- 9 and executioner caspase- 3 . Active caspase- 3 cleaves ICAD, releasing and allowing nuclear translocation of $C A D$, the main apoptotic endonuclease, triggering apoptotic DNA degradation. Alternatively, apoptotic stimuli induce nuclear translocation of AChE-S (3), which can directly hydrolyze DNA. An attractive hypothesis is that AChE could act as a "waste-management" DNase, as long as the secretion pathway of AChE-S functions in injured non-cholinergic tumoral cells. AChE-S, synaptic acetylcholinesterase; CAD, caspase-activated DNase; ICAD, inhibitor of CAD; TFs, transcription factors. nuclease activity of AChE-S is optimal at physiological $\mathrm{pH}$ (around 7.5) and generates 3' OH-free TUNEL-positive DNA ends. Interestingly, DNase I also shares these biochemical features (reviewed in [1]). Du and colleagues also demonstrate that, unlike DNase I, AChE-S is not inhibited by G-actin, a general and abundant cytoskeleton component often found in extracellular media after plasma membrane breakdown following, for example, necrotic cell death during inflammatory processes. Therefore, it is intriguing to speculate that the DNase activity of AChE-S might occur not only inside but also outside the cell, mimicking DNase I task in a G-actin-enriched extracellular environment.

\section{María Sánchez-Osuna ${ }^{1}$, Victor J Yuste ${ }^{1}$}

${ }^{l}$ Cell Death, Senescence and Survival group, Departament de Bioquímica i Biologia MolecularUnitat de Medicina \& Institut de Neurociències, Facultat de Medicina, Universitat Autònoma de Barcelona, 08193 Barcelona, Spain Correspondence: Victor J Yuste E-mail: victor.yuste@uab.cat

\section{References}

1 Samejima K, Earnshaw WC. Nat Rev Mol Cell Biol 2005; 6:677-688.

2 Higuchi Y. Biochem Pharmacol 2003; 66:1527-1535.

3 Widlak P, Garrard WT. J Cell Biochem 2005; 94:1078-1087.

4 Aleksandrushkina NI, Vanyushin BF. Biochemistry 2012; 77:1436-1451.

5 Festjens N, Vanden Berghe T, Vandenabeele P. Biochim Biophys Acta 2006; 1757:13711387.

6 Du A, Xie J, Guo K, et al. Cell Disc 2015 Apr 28. doi:10.1038/celldisc.2015.2

7 Zhang XJ, Greenberg DS. Front Mol Neurosci 2012; 5:40.

8 Iglesias-Guimarais V, Gil-Guinon E, Sanchez-Osuna M, et al. J Biol Chem 2013; 288:9200-9215.

9 Zhao Y, Wang X, Wang T, et al. Hepatology 2011; 53:493-503.

10 Kumar A, Singh A, Ekavali. Pharmacol Rep $2015 ; 67: 195-203$.

11 Zhang JY, Jiang H, Gao W, et al. Cell Mol Life Sci 2008; 65:1435-1445.

12 Gao W, Zhu H, Zhang JY, Zhang XJ. Cell Mol Life Sci 2009; 66:2181-2193. 